\title{
PREFACE
}

\section{History of the Development of the Method of Content Analysis}

The method of verbal content analysis presented in the fol. lowing chapters had antecedents in earlier work of the authors. Even prior to this collaborative endeavor, the senior author had committed himself to the investigation of verbal behavior.

While a research associate at the Institute for Psychosomatic and Psychiatric Research and Training of the Michael Reese Hospital in Chicago, Illinois (1948-1951), Dr. Gottschalk became interested in exploring emotional factors that contribute to or trigger epileptic convulsions. It was during the process of these psychophysiological studies of epilepsy that he decided to pursue the systematic and microscopic analysis of verbal behavior. Briefly, the sequence of events that aroused his interest was the following. $\mathrm{He}$ took three boys into psychotherapy; one, 17 years old, received psychoanalytic psychotherapy, and the other two, $41 / 2$ and 8 years old, received child psychoanalysis. The results of this study (Gottschalk, 1953, 1956) provided strong evidence that psychological factors played a causal role in the occurrence of the epileptic seizures of these children. On moving to the National Institute of Mental Health at Bethesda, Md., in June, 1951 to become a research psychiatrist there, Dr. Gottschalk decided to pursue further the psychophysiological factors in epilepsy. Dr. David McK. Rioch, Director of the Division of Psychiatry and Neurology, Post-Graduate School at the Walter Reed Army Institute of Research, made available to Dr. Gottschalk rich clinical material and provided electroencephalographic laboratory facilities. Several longitudinal investigations were begun of military personnel who had developed seizures during their tour of duty. Patients were selected for study who had frequent discrete paroxysms (about one every 2-3 minutes) of readily distinguishable high- 
amplitude fast or slow waves in their electroencephalograms. The choice of subjects was based on the hope that it might be possible to relate the acute arousal of emotions and psychological conflicts to the abnormal, high-amplitude, paroxysmal brain waves. The experimental procedure that was used consisted of having patients free-associate while recording their electroencephalogram, electrocardiogram, electromyogram of gross muscular tension of upper and lower extremities, and galvanic skin response. The patients' free associations were tape-recorded and synchronized with the electrical findings. From these studies Dr. Gottschalk was able to make the observation that speaking as well as doing silent mental arithmetic tended to suppress the frequency of paroxysmal electroencephalographic activity and that the arousal of separation anxiety appeared to trigger many of the epileptic seizures of the patients (Gottschalk, 1955). He was dissatisfied, however, with the precision with which he could measure the intensity of separation anxiety or other affects of possible relevance to the abnormal, high-amplitude brain-wave activity from the patients' recorded free associations. It was, in fact, the problems encountered in measuring emotions and other psychological states from typescripts of speech obtained in the studies that led Dr. Gottschalk to resolve to pursue further the content analysis of speech.

At the National Institute of Mental Health he now teamed up with a long-time friend and fellow psychiatrist and psychoanalyst, Dr. Gove Hambidge, Jr., to pursue methods of analyzing and measuring communication processes. The two men explored a number of ways of eliciting and recording different channels for the communication of affects and other psychological states, for example, by lexical features in speech, by paralanguage features (vocalizations, voice quality, voice set), by linguistic features (junctures, stress, pitch), and by kinesic features (posture and gestures) of behavior. They studied tape recordings of speech obtained under different circumstances and also $16 \mathrm{~mm}$. movies of action sequences. Although both of them were accustomed, as clinicians, to using cues from all possible channels of communication as to how a person was feeling and what he was thinking, they finally decided that it was impossible for them to analyze and note systematically all the elements in all these communication channels and to determine how quantitative as well as quali- 
tative aspects of psychological states were communicated separately and conjointly through these different channels. They decided to narrow and focus their attention on characteristics of speech, especially lexical content features. They devised two standardized techniques of eliciting speech, both attempting to maximize the human tendency toward projection of intrapsychic qualities, response sets, and attitudes. The verbal method of eliciting speech used instructions which told subjects that this was a study of speaking and conversational habits and asked the subjects to talk for five minutes about any interesting or dramatic personal life experience they had ever had. The visual method of induction of speaking involved asking subjects to tell stories for five minutes about certain pictures from Murray's Thematic Apperception Test (1943). Two types of analysis of the speech were carried out: (1) form analysis, which involved such variables as rate of speech, frequency and duration of pauses, and frequency of "fills" (uhs, ahs, ers), of incomplete words, of sequential repetitions, of nonlexical vocalizations (laughs, coughs, sneezes, throat clearings, sniffles, etc.), and of aposiopeses (instances in which the speaker suddenly breaks off the expression of a thought and introduces another); and (2) content analysis, which involved a consideration of the meaning, relationships, objects, concepts, and processes symbolized by the verbal elements in speech. Content analysis was at the level of single words rather than groups of words or themes. Every word was categorized by a system of codification of language largely derived from psychodynamic principles, and this classification was labeled a "psychological" one. A second system of classification of the words was a grammatical one.

The "psychological" system of classification was as follows. One class of words included all those which referred to environmental objects. These in turn differentiated into self and nonself. Nonself was broken down into other human objects, animals, flora, and inanimate objects. A second class of words was concepts or abstractions. A third class included subgroups of words expressing neuromuscular actions, motivation, perceptions, and thought, all of which represented types of processes between objects or concepts. Other word classes included references to measure, qualification, gender, part-whole differentiations, and negations. 
Measure references consisted of words indicating some relativity of time, space, or quantity regardless of part of speech or context in which used.

The grammatical classification included adjectives, adverbs, nouns, pronouns, verbs, prepositions, conjunctions, and interjections.

The first paper published from these early investigations (Gottschalk and Hambidge, 1955) indicated that the methods of eliciting speech (verbal and visual) evoked different percentages of these categories of words from the same individual and that certain patterns of words in five-minute verbal samples obtained after psychoanalytic sessions with two patients were associated with recurring focal conflicts occurring during the psychoanalytic sessions.

The next study involved an examination and comparison of the differences in the form and content of the speech patterns of a small group of psychotic and nonpsychotic individuals. Differences were found between the two groups in both form and content of five-minute verbal samples elicited by the so-called verbal method. Formwise, the psychotic subjects showed a decreasing word rate per minute as compared to the nonpsychotic subjects. Contentwise, the psychotic patients showed a greater number of references to the self and to negations; greater use of verbs; lesser use of "we" and "us," of words referring to inanimate objects, and of qualifying words; and a significantly smaller use of words which denote or connote location or spatial relationship (regardless of grammatical use) as compared to the nonpsychotic subjects (Gottschalk et al., 1957). ${ }^{1}$

In another study carried out about this time (Hambidge and Gottschalk, 1958), 80 verbal samples were obtained from a single subject on a once-a-week basis over a period of a year while this individual underwent a psychoanalysis. The verbal samples were all obtained by the psychoanalyst (G.H.) at a regular time following one of the psychoanalytic sessions. Forty of the 80 verbal samples were elicited by the "visual" method, which simulates the projective test situation, and 40 were elicited by the "verbal"

1 This data, although collected in 1952-1953 by Dr. Gottschalk while at the National Institute of Mental Health, Bethesda, Maryland, was not fully analyzed and prepared for publication until 1956, after Dr. Gottschalk had moved to Cincinnati; Dr. Gleser assisted in the structuring and statistical analysis of the data. 
method, which simulates the psychiatric interview situation. Again evidence was obtained supporting the hypothesis that the choice and frequency of use of certain words in the verbal samples were related to the dynamic state of the patient both quantitatively and qualitatively, as observed in analytic treatment sessions. Consistent differences were found between verbal samples obtained in the projective test situation (visual induction of speech) and psychiatric interview situation (verbal induction of speech). Further evidence was obtained which indicated that the psychoanalytic experience and the state of therapeutic progress at the time of giving the verbal sample influence the way a patient expresses himself in terms of the choice and use of language variables in the visually induced and verbally induced verbal samples.

The last two studies terminated the collaboration of Louis Gottschalk and Gove Hambidge, Jr., for Dr. Gottschalk moved in September, 1953, to Cincinnati, Ohio, to become a faculty member of the Department of Psychiatry, University of Cincinnati, College of Medicine.

Gradually Dr. Gottschalk made further efforts to explore the use of content features in language as a possible tool for the quantitative analysis of psychological states; for the first time, an effort was made to examine thematic content categories, using the grammatical clause instead of a word as the unit of communication. It was found in a double-blind drug-placebo study that a psychoactive pharmacological agent, pipradrol-a medicament similar in action to dextro-amphetamine-significantly increased thematic references to achievement strivings (Gottschalk et al., 1956). In another study, a very complex psychological position (a masochistic problem-solving approach to obtaining support and love) was found to be significantly associated with the percentage of streptococcal bacteria that could be cultured from the oropharynx of a woman with rheumatic heart disease (Kaplan, Gottschalk, and Fleming, 1957). A cross-validation study tended to confirm this hypothesis by finding a significant correlation $(0.40, p<.05)$ between a frequency count of relevant themes in 20 five-minute verbal samples and the relative percentage of streptococcal bacterial colonies cultured from the patient's throat (Gottschalk and Kaplan, 1958b; Kaplan and Gottschalk, 1958).

In 1955, Dr. Gottschalk obtained a U.S. Public Health Serv- 
ice research project grant enabling him to pursue verbal behavior studies on a broader scale. At this point he met Dr. Goldine C. Gleser, a psychologist from St. Louis, Mo., with extensive experience in interdisciplinary research and mathematical statistics, and he invited her to collaborate with him. They decided to study speech at both the "atomistic" level (words) and "molecular" level (themes). A first step taken was to obtain five-minute verbal samples from 90 "normative" individuals, all medically healthy and capable of gainful employment. The group was stratified for sex and intelligence. From this investigation came a contribution to the "atomistic" approach to content analysis, giving in detail the relationship of sex and intelligence to the choice of words (Gleser, Gottschalk, and John, 1959). Data obtained from the study were also used in a number of reports featuring the thematic approach to content analysis (Gleser et al., 1961; Gottschalk et al., 1960a, 1963; Gottschalk and Gleser, 1964a).

The atomistic approach was applied to a set of false and genuine suicide notes collected by Shneidman and Farberow (1957) in an investigation to determine factors contributing to suicide. Analysis of the notes yielded a set of characteristics by which the genuine suicide notes could be distinguished from the pseudosuicide ones. The criteria of differentiation were further substantiated by applying them to another set of genuine suicide notes obtained from the Cincinnati Police Department. The practical application of the distinctive features of the genuine suicide notes to the problem of ascertaining whether any suicide note was a genuine or false one was illustrated using a decision model. For example, it was observed that, if those notes were considered genuine in which the percentage of auxiliary words minus the percentage of references to others (second and third person) is less than 14 percent plus those remaining notes in which the percentage of references to inanimate objects is greater than 1 percent (Gottschalk and Gleser, 1960a), it would be possible to identify successfully 94 percent of the genuine suicide notes while misidentifying only 15 percent of the false ones.

The suicide note study marked the last of the published reports using the atomistic approach to content analysis. The ensuing period of research marked the beginning of the systematic development of verbal behavior measures of transient affects, such as anxiety and hostility, at the level of thematic content. Dr. 
Gottschalk and Dr. Gleser worked toward evolving principles whereby the specification and quantification of psychological states could be derived from brief samples of a subject's speech. Reliability and construct validation studies were carried out with different samples of subjects under different treatment situations over a period of many years. The Public Health Service Research Project Grant to pursue these verbal behavior studies was renewed in 1957, and financial support has continued to the present time from one or another division of the National Institute of Mental Health. Other sources of research funds (Public Health Service, M-1055, M-6005, MH-08282, K3-MH-14,665; Foundations Fund for Research in Psychiatry, T57-74; National Association for Mental Health; Veterans Administration; Schweppe Research and Education Foundation; Defense Atomic Support Agency Contract \#Da-49-146-xz-315; Robert D. Stern Fund) were obtained to help support continuing investigation and development of this content analysis method. An exposition and elaboration of these studies and the method constitute the subject matter of this book. The theoretical underpinnings, reliability studies, construct validation studies, "normative" and demographic data for various scales, applications (and further construct validation studies) in the areas of research in psychotherapy, psychoanalysis, psychophysiology, psychosomatic medicine, psychopharmacology, psychology of affects, prediction of outcome with various treatments, and so forth, are covered in considerable detail. Finally, a glimpse is provided into future directions and possible applications of the content analysis method. 
